\title{
TG20
}

\section{Integrated Petrophysical Characterization of Tight Gas Prospects}

\author{
D. Jacobi (Baker Hughes), D. Georgi* (Baker Atlas) \& B. LeCompte (Baker \\ Hughes)
}

\section{SUMMARY}

Commercial success and successful completion of tight gas prospects requires an understanding of the complexities of the rock matrices which can either promote or curtail production. Porosity, occluded porosity, variations in grain size, the total organic carbon, the formation of clay minerals in pore spaces, and the recognition of open or mineralized fractures, as well as seals and compartmentalization within the strata must be determined to assess potential productivity and to develop effective completion strategies. Often there is an absence of petrophysical models that can account for or predict and distinguish these reservoir properties and a dearth of formation evaluation data.

Conventional formation evaluation measurements and conventional interpretation techniques are challenged when applied to tight gas reservoirs. However, the integration of a host of new technologies can significantly aid in the evaluation of gas shales and tight gas reservoirs, resulting in significant improvement and reduction in risk for development of tight-gas plays. With the use of newly developed geochemical sondes, nuclear magnetic resonance interpretations, acoustic compressional and shear velocities, borehole resistivity images and deep reading acoustic images, we can obtain accurate porosities, mineralogy, and total-organic carbon, gas, and water saturations and map fractures away from the borehole. Further, these measurements can be used to differentiate between lithofacies which either influence or curtail gas production and to compute critical geomechanical properties that predict which facies are favorable for hydraulic fracture stimulation and which might be frac barriers. The integrated petrophysical method has the potential to reduce completion cost and enhance production for vertical well prospects and provide valuable direction for drilling horizontal wells in gas shales and tight gas sand reservoirs. 